\title{
REPRESENTATION OF $C^{n}$-OPERATORS
}

\section{S. KANTOROVITZ ${ }^{1}$ AND K. J. PEI}

ABSTRACT. The operator $T_{n}=M+n J$ acting in $C(I)$, where $I=$ $[0,1], M: f(x) \rightarrow x f(x)$, and $J: f(x) \rightarrow \int_{0}^{x} f(t) d t$, is known to be of class $C^{n}$ (cf. [2], [3], [4]). We show here that every real operator of class $C^{n}$ in a weakly complete Banach space $X$ has a "weak representation" as $T_{n}$.

1. Weak representation of $C^{n}$-operators. We need the following extension of [2, Lemma 2.8] to weakly complete spaces.

Lemma 1. Let $X$ be a weakly complete Banach space, and let $T$ be a $C^{n}$-operator (i.e., an operator of class $C^{n}$ ) in $X$, with spectrum in $I$. Then the $C^{n}(I)$-operational calculus $T(\cdot)$ for $T$ is uniquely representable in the forin

$$
T(f)=\sum_{j=0}^{n-1} f^{(j)}(0) T^{j} / j !+\int_{I} f^{(n)}(s) F(d s),
$$

$f \in C^{n}(I)$, where $F$ is a uniformly bounded operator measure on the Borel subsets of $I$ ( $F$ is strongly countably additive and the integral in (1) is understood in the strong operator topology). As usual, $\sum_{j=0}^{-1} a_{j}=0$ for any $a_{j}$.

Proof. For $f \in C^{n}(I)$, we have

$$
f(x)=\sum_{j=0}^{n-1} f^{(j)}(0) x^{j} / j !+\left(J^{n} f^{(n)}\right)(x),
$$

hence

$$
T(f)=\sum_{j=0}^{n-1} f^{(j)}(0) T^{j} / j !+T\left(J^{n} f^{(n)}\right) .
$$

When $f$ ranges in $C^{n}(I), f^{(n)}$ ranges in the entire space $C(I)$, and it follows

Received by the editors December 11, 1973. $47 \mathrm{~B} 40$.

AMS (MOS) subject classifications (1970). Primary 47 A65, 47 A60; Secondary

Key words and phrases. $C^{n}$-operators, representation, operator measure, characteristic operator.

${ }^{1}$ Research supported by NSF Grant No. GP-24917A \#1. 
that for each $y$ in $X$, the map $U_{y}: f^{(n)} \rightarrow T\left(J^{n} f^{(n)}\right) y$ is a bounded linear operator from $C(I)$ into $X$, and it is necessarily weakly compact since $X$ is weakly complete (cf. [1, Theorem VI.7.6]). By Theorem VI.7.3 in [1], there exists a unique vector measure $m_{y}$ on the Borel sets in $I$, countably additive and uniformly bounded by $\left\|U_{y}\right\| \leq K\|y\|$, such that

$$
U_{y}(g)=\int_{I} g(s) m_{y}(d s), \quad g \in C(I)
$$

For each Borel subset $\delta$ of $I$, write $F(\delta) y=m_{y}(\delta)$. Then $F(\delta) \in B(X)$, $\|F(\delta)\| \leq K$, and the Lemma follows easily from (2) and (3).

By the final remark in [5], we have the following

Corollary. A real operator on $l^{1}$ is singular of class $C^{n}$ iff it is spectral of type $n$.

(See [2] for the terminology.)

Definition. The measure $F$ in Lemma 1 will be called the characteristic measure of $T$, and the map $U: f \rightarrow \int_{I} f d F$ of $C(I)$ into $B(X)$ is then called the characteristic operator of $T$.

Definition. A weak representation for the $C^{n}$-operator $T$ is a bounded operator $U: C(I) \rightarrow B(X)$ such that

$$
T(f) U h=U T_{n}(f) h
$$

for all $f$ in $C^{n}(I)$ and $h$ in $C(I)$, where $T(\cdot)$ and $T_{n}(\cdot)$ are the operational calculi for $T$ and $T_{n}$ respectively.

Theorem 1. Let $T$ be a $C^{n}$-operator with spectrum in 1 , acting in a weakly complete Banach space. Then the characteristic operator of $T$ is a weak representation for $T$.

Proof. Since both $T$ and $T_{n}$ are of class $C^{n}(I)$ and polynomials are dense in $C^{n}(I)$, it suffices to verify that

$$
T^{k} U=U T_{n}^{k}, \quad k=0,1,2, \cdots,
$$

which in turn follows readily from the special case with $k=1$.

By (1), we have $T\left(J^{n} h\right)=U h, h \in C(I)$. Hence, by Lemma 1 in [3], we have for all $h \in C(I)$ :

$$
U T_{n} h=T\left(J^{n} T_{n} h\right)=T\left(M J^{n} h\right)=T\left(x J^{n} h\right)=T(x) T\left(J^{n} h\right)=T U h .
$$


Remark. The reader will realize by now that in (4) one has a composition of operators on the right side and the multiplication of the operators $T(f)$ and $U h$ on the left side. For $n=0$, (4) reduces to the multiplicativity property of $T(\cdot)=U$.

Theorem 2. Let $T$ be a bounded linear operator on the (arbitrary) Banach space $X$, and let $n$ be a nonnegative integer. Suppose there exists a bounded operator $U: C(I) \rightarrow B(X)$ such that

(i) $U 1=T^{n} / n$ ! and

(ii) $T U=U T_{n}$.

Then $T$ is of class $C^{n}(I)$ and $U$ is its characteristic operator.

Corollary. Let $T$ be a bounded linear operator on the weakly complete Banach space $X$, and let $n$ be a nonnegative integer. Then $T$ is of class $C^{n}(I)$ iff there exists a bounded linear operator $U: C(I) \rightarrow B(X)$ satisfying (i) and (ii).

Proof. Define $T(\cdot)$ on $C^{n}(I)$ by

$$
T(f)=\sum_{j=0}^{n-1} f^{(j)}(0) T^{j} / j !+U f^{(n)}, \quad f \in C^{n}(I) .
$$

It suffices to verify that

$$
T\left(x^{m}\right)=T^{m} \quad \text { for all } m=0,1, \cdots .
$$

This is trivial for $m<n$, and it remains to show that

$$
T\left(x^{n+k}\right)=T^{n+k}, \quad k=0,1, \ldots .
$$

Since $T\left(x^{n+k}\right)=((n+k) ! / k !) U x^{k}$ by (5), we must show that

$$
U x^{k}=k ! T^{n+k} /(n+k) !, \quad k=0,1, \cdots .
$$

This is done by induction. For $k=0$, (7) reduces to (i). Assuming (7) for $k$ and using (ii), we obtain

$$
U x^{k+1}=\frac{k+1}{n+k+1} U T_{n} x^{k}=\frac{k+1}{n+k+1} T U x^{k}=\frac{(k+1) !}{(n+k+1) !} T^{n+k+1} .
$$

Combining Theorems 1 and 2, we obtain the corollary.

2. Characteristic measures. Let $F$ be a (weakly, hence strongly, countably additive) operator measure on the Borel sets of $I$, and let $U: C(I) \rightarrow$ $B(X)$ be defined by $U f=\int_{I} f d F$. Let $M_{k}$ be the kth moment of $F, M_{k}=U\left(x^{k}\right)$, 
and denote by $c_{\delta}$ the characteristic function of the set $\delta$.

Theorem 3. F is the characteristic measure of a $C^{n}(I)$-operator if and only if it satisfies the "characteristic identity"

$$
F(\delta) F(\epsilon)=\sum_{k=0}^{n}\left(\begin{array}{l}
n \\
k
\end{array}\right) U\left(J^{k} c_{\delta} J^{n-k} c_{\epsilon}\right)
$$

for all Borel subsets $\delta, \epsilon$ of $I$, and (in case $n \geq 2$ ) there exists an operator $T$ such that

$$
T^{n+k}=(n+k) ! M_{k} / k !, \quad k=0, \cdots, n-1 .
$$

When this is the case, $F$ is the characteristic measure of the operator $T$.

Remark. For $n=0$, (8) reduces to the spectral measure condition $F(\delta) F(\epsilon)=F(\delta \cap \epsilon)$. For $n=1$, the "coherence condition" (9) becomes $T=$ $M_{0}$, which is just a definition of $T$.

Proof. Necessity. Suppose $F$ is the characteristic measure of the $C^{n}(I)$ operator $T$. Then for all $f, g$ in $C(I)$,

$$
U f U g=T\left(J^{n} f\right) T\left(J^{n} g\right)=T\left(J^{n} f J^{n} g\right)=U\left(\left(J^{n} f J^{n} g\right)^{(n)}\right),
$$

and so by Leibnitz' formula

$$
U f U g=\sum_{k=0}^{n}\left(\begin{array}{l}
n \\
k
\end{array}\right) U\left(J^{k} f J^{n-k} g\right)
$$

for all $f, g$ in $C(I)$, and hence for all bounded Borel functions $f, g$ on $I$. Taking, in particular, characteristic functions, (8) follows.

For any $k \geq 0, x^{n+k} \in C_{0}^{n}(I)=\left\{f \in C^{n}(I) ; f^{(j)}(0)=0, j=0, \cdots, n-1\right\}$; hence

$$
T^{n+k}=T\left(x^{n+k}\right)=U\left(\left(x^{n+k}\right)^{(n)}\right)=(n+k) ! M_{k} / k ! \text {. }
$$

Sufficiency. The case $n=0$ being well known, we assume $n \geq 1$. By linearity, it follows from (8) that $(10)$ is valid for simple Borel functions, and hence for all $f, g$ in $C(I)$. Writing $u=J^{n} f, v=J^{n} g$ in (10), we obtain

$$
U u^{(n)} U v^{(n)}=U\left((u v)^{(n)}\right)
$$

for all $u, v$ in $J^{n} C(I)=C_{0}^{n}(I)$.

Let $T$ be the operator involved in the coherence condition (9), and define $T(\cdot)$ as in (5). Again, it suffices to verify (6) for $m \geq n$. For $n \leq m \leq$ $2 n-1,(6)$ follows from (5) and (9). For $m \geq 2 n$, write $m=q n+r$ uniquely 
with $q \geq 2$ and $r<n$, then represent $x^{m}$ as a product of $q C_{0}^{n}(I)$-functions $x^{m}=x^{n+r}\left(x^{n}\right)^{q-1}$. By (5) and (11), $T(\cdot)$ is multiplicative on $C_{0}^{n}(I)$. Since (6) is valid for $m<2 n$, we obtain

$$
T\left(x^{m}\right)=T\left(x^{n+r}\right)\left(T\left(x^{n}\right)\right)^{q-1}=T^{n+r} T^{n(q-1)}=T^{m}
$$

as wanted.

Corollary. $F$ is the characteristic measure of a $C^{n}$-operator with spec. trum in $R^{+}=(0, \infty)$ iff $(8)$ holds, $\sigma\left(M_{0}\right) \subset R^{+}$, and in case $n>2$,

$$
\left(n ! M_{0}\right)^{n+k}=\left[\frac{(n+k) !}{k !} M_{k}\right]^{n}, \quad k=1, \cdots, n-1 .
$$

Proof. The necessity part follows trivially from the corresponding part in Theorem 3.

For the converse, note that for any operator $S$ with spectrum in $R^{+}$and for any real $\alpha, s^{\alpha}$ may be defined by means of the analytic operational calculus, and the composite function theorem implies that $\left(S^{\alpha}\right)^{\beta}=\left(S^{\beta}\right)^{\alpha}=S^{\alpha \beta}$ ( $\alpha, \beta$ real). Define $T=\left(n ! M_{0}\right)^{1 / n}$. Then $\sigma(T) \subset R^{+}$, and $\sigma\left(M_{k}^{n}\right) \subset R^{+}, k=0$, $\cdots, n-1$ (by (12)). Hence, by (12) and the composite function theorem for the analytic operational calculus,

$$
T^{n+k}=\left[\left(n ! M_{0}\right)^{n+k}\right]^{1 / n}=\frac{(n+k) !}{k !}\left(M_{k}^{n}\right)^{1 / n}=\frac{(n+k) !}{k !} M_{k},
$$

and the conclusion follows from Theorem 3.

\section{REFERENCES}

1. N. Dunford and J. T. Schwartz, Linear operators. I: General theory, Pure and Appl. Math., vol. 7, Interscience, New York, 1958. MR 22 \#8302.

2. S. Kantorovitz, Classification of operators by means of their operational calculus, Trans. Amer. Math. Soc. 115 (1965), 194-224. MR $33 \# 7855$.

3., The $C^{k}$-classification of certain operators in $L_{p}$, Trans. Amer. Math. Soc. 132 (1968), 323-333. MR 37 \#2033.

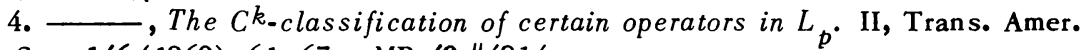
Math. Soc. 146 (1969), 61-67. MR 40 \#4814.

5. B. Walsh, Spectral decomposition of quasi-Montel spaces, Proc. Amer. Math. Soc. 17 (1966), 1267-1271. MR 34 \#4914.

DEPARTMENT OF MATHEMATICS, UNIVERSITY OF ILLINOIS AT CHICAGO CIRCLE, CHICAGO, ILLINOIS 60680 (Current address of S. Kantorovitz)

DEPARTMENT OF MATHEMATICS, BAR-ILAN UNIVERSITY, RAMAT-GAN, ISRAEL

Current address (K. J. Pei): Department of Mathematics, Brooklyn College (CUNY), Brooklyn, New York 11210 